\title{
Optimization of English Learning Platform Based on a Collaborative Filtering Algorithm
}

\author{
Jiali Tang \\ School of Foreign Languages of Zunyi Medical University, Zunyi, Guizhou 563000, China \\ Correspondence should be addressed to Jiali Tang; tjl@zmu.edu.cn
}

Received 22 December 2020; Accepted 23 April 2021; Published 30 April 2021

Academic Editor: Wei Wang

Copyright ( $\odot 2021$ Jiali Tang. This is an open access article distributed under the Creative Commons Attribution License, which permits unrestricted use, distribution, and reproduction in any medium, provided the original work is properly cited.

\begin{abstract}
This paper provides a detailed description of the recommendation system and collaborative filtering algorithm to optimize the English learning platform through the collaborative filtering algorithm and analyses the algorithmic principles and specific techniques of collaborative filtering. After introducing the recommendation system and collaborative filtering algorithm, this paper elaborates on the theoretical basis and technical principles of the recommendation algorithm based on cognitive ability and difficulty and provides an in-depth analysis of the design and implementation of the recommendation algorithm by combining cognitive diagnosis theory, readability formula, and English knowledge map, which provides a comprehensive and solid theoretical guidance and support for the application development of the online English learning platform. The system is tested by building a Spring Cloud platform, importing actual business data, focusing on the validation of the recommendation model, and connecting the recommendation system to the formal production system to analyse the recommendation effect. Compared with the original recommendation method, the online English learning platform designed and implemented in this paper based on the cognitive ability and difficulty collaborative filtering recommendation algorithm has a better recommendation effect. The system is proved to be well designed and has certain reference and guiding value for the whole web-based online learning platform and has a broader application prospect nowadays and in the future.
\end{abstract}

\section{Introduction}

With the birth of computers, human society has entered the information age and the network age, and what was thought to be a huge human society has become a "global village," and with the export of British culture, the people in the global village have tended to assimilate in terms of language communication, and English has become the main carrier of information exchange among the villagers [1]. English is now the most widely spoken language among the villagers [2]. The development of the Internet has facilitated the further spread of English, and web-based English learning platforms have been created to better facilitate English learning [3]. The research in this paper is centred on how to promote online English learning platforms for better English learning. A new Internet learning and education model that can exclude the difficulties of time, space, and location and bring the excellent teachers all over the world, closer to the students, so that every student can enjoy the best education is live Internet education [4]. While adults obtain workplace courses through the Internet and junior and senior high school students conduct extracurricular training and tutoring through the Internet, the market of children's education has also ushered in a hot development stage in recent years, because the best stage of education is always the earliest stage [5]. In recent years, several online learning platforms for cybersecurity have emerged, and these learning platforms have been accepted and recognized by most cybersecurity learners [6]. However, it should be noted that existing cybersecurity online learning platforms are still in their infancy, and there are still many problems that need to be solved [7]. For example, the existing cybersecurity 
online learning platform is more like an unmanned supermarket full of various learning resources, which is limited to the collection and sharing of learning materials but does not have a suitable shopper to help learners select the learning resources suitable for their own needs [8]. More learning resources and more data are not necessarily a good thing for learners. Especially for the clueless beginner, it can be a disaster [9]. It is a needle in a haystack to find the right learning path and materials for the learner, which will eventually lead to a complete loss of interest and motivation [10].

Margaret believes that the blended learning model has a very rich connotation and broadly covers aspects including four points: first, the adoption of multiple networked technologies to achieve educational goals; second, the combination of teacher-led training and modern teaching techniques; third, the use of modern teaching techniques to accomplish teaching tasks; and fourth, the full use of multiple teaching methods and the use of advanced teaching techniques to ultimately achieve optimal learning outcomes [11]. Li argues that this new learning model is to make full use of modern teaching techniques to meet individual learning styles and thus achieve personalized customization of teaching to achieve the best teaching results [12]; Liu and $\mathrm{Hu}$ argue that there are major differences between the two models of traditional learning and blended learning and that the former needs to go through five models in the process of transformation to the latter [13]. They are the complementary model, the central market model, the cafeteria model, the replacement model, and the fully online model [14]; Fan argue that the blended learning model is a combination of distance education and face-to-face teaching, which does not require the use of web pages for teaching in the classroom but maximizes the integration of face-to-face and online learning to fully combine the advantages of each [15]; according to Rehm, adopting a blended learning model means using various advanced technologies to integrate the desired goals [16]; Li proposed a "5R definition," in which they considered that a blended learning model combines five aspects, which are technology, object, knowledge, and skills, time, and learning style, under which the best teaching and learning results are achieved [17]. Weeks investigated the blended learning model and from the findings, this learning model can show different effects depending on the disciplinary differences in learning; this finding has triggered a wide range of scholarly attention [18]; Hwang and $\mathrm{Fu}$ constructed a model to study the relationship between internal student satisfaction and needs to be based on a blended learning environment to perceive learning outcomes and motivation [19]. Shuo and Xiao studied the social networks of university students in a blended learning environment and analysed the impact of perceptions and levels of knowledge construction on actual teaching and learning [20]. Liang explored usability factors in a blended learning environment and studied the impact of external support on the use of the Moodle platform by university students [21].

Firstly, the diverse characteristics of English teaching in high school are fully considered, and a new blended learning model that conforms to students' learning rules is constructed and applied to English teaching from practice. Secondly, we investigate how the blended learning model based on Sequoia Smart Learning Platform can improve students' English learning efficiency, how students develop an interest in English learning, the degree of students' independent learning, and their acceptance of the blended learning model based on Sequoia Smart Learning Platform. Finally, follow-up questionnaires and interviews were held with students and teachers to understand students' acceptance of the blended learning model based on Sequoia Smart Learning Platform and to summarize the advantages and disadvantages of the existing teaching model. The network security independent learning platform developed in this paper solves the problems of existing network security online learning platforms by introducing personalized recommendation algorithms [11]. The platform actively mines learners' learning interests and learning preferences, filters other irrelevant content, and provides learners with accurate personalized recommendations by using the userbased collaborative filtering recommendation algorithm proposed earlier, so that information valuable to learners (such as learning routes and learning resources) can be recommended to that learner. This saves learners' time and improves their learning experience and learning efficiency. On the other hand, it also improves learners' recognition and reliance on the platform and establishes a long-term and stable relationship between the platform and learners, thus laying the foundation for better access to learner-related data later. This paper researches personalized recommendation technology proposes a new user-based collaborative filtering recommendation algorithm and applies the algorithm to the network security self-learning platform, so that it can help each learner find the learning resources they need as soon as possible and improve the learner's learning interest and learning efficiency. The work in this paper is of great value in both theoretical research and practical application, especially in improving the quality of cybersecurity personnel training.

\section{English Optimization Design of a Collaborative Filtering Algorithm}

\subsection{Improved Collaborative Filtering Algorithm.} Collaborative filtering is to recommend to users their favourite things based on similar preferences. Specifically, if two users rate some items roughly the same, they may also rate other items roughly the same; if most people have similar preferences for some items, then an unknown user will have similar preferences for those items. Many collaborative filtering algorithms use the nearest neighbour technique; i.e., they use some computational method to calculate the similarity between users. Therefore, the core of these collaborative filtering algorithms is the similarity calculation, which calculates the similarity between users or items to predict the ratings of items, and finally, the $N$ items with the highest ratings are displayed to the users. The recommendation system looks for patterns in users' selection behaviours and preferences identify users with similar preferences and make recommendations based on similar users [22]. The user preference information collected by the 
system will play a decisive role in the effectiveness of the system's recommendations. Since there are many kinds of information that users can provide to the system, it is a prerequisite for the success of a recommendation system to select the user preference information that is critical to the recommendation and to preprocess this user preference information effectively. Usually, the preprocessing of user information includes grouping or weighting user information to obtain a two-dimensional rating matrix reflecting user preferences, where the columns of the matrix are a list of users and the rows are a list of items, and each rating data is a user's preference for a certain item, as shown in Figure 1.

Calculating the similarity of users is the key step of the collaborative filtering recommendation algorithm, in which we need to calculate the similarity between different users first. Based on the result of similarity calculation, the $k$ nearest neighbours of the target user can be determined (i.e., the $k$ users that are most like the target user are the nearest neighbours of the target user). In the user-item matrix, the user's personal rating preferences influence the magnitude of the rating value [23]. In the process of similarity calculation, the variability of the values will represent the variability of the similarity calculation results. The classical cosine similarity does not consider the different rating scales of different users; for example, in the case of rating range $1-5$, for user A, a rating above 3 is his favourite, while for user $B$, a rating above 4 is his favourite. For the above problem, it is necessary to extend the classical cosine similarity to avoid the problem that different users have different scales of ratings. For the above problem, a commonly accepted approach is to use "modified cosine similarity" to calculate the similarity between users. The modified cosine similarity can effectively solve the problem that different users have different rating scales by subtracting the average rating of the items.

$$
\operatorname{sim}(x, y)=\frac{\sum_{i \in I_{x, y}}\left(R_{x i}-R_{i}\right) \times\left(R_{y i}-R_{i}\right)}{\sqrt{\sum_{i \in I_{x, y}}\left(R_{x i}-R_{i}\right)_{i}^{2} \times\left(R_{y i}-R_{i}\right)_{y}^{2}}} .
$$

After calculating the similarity of any two users, the set $N$ of nearest neighbours of the target user can be found, and then the Top- $N$ model can be used to recommend items for the target user. First, the set $N$ of nearest neighbours of the target user $x$ is obtained based on some similarity calculation method; second, the elements in the set $N$ are sorted according to the predicted ratings to obtain the set of columns of Top- $N$; finally, the predicted values of the ratings of the target user $x$ are calculated using the Top- $N$ set with the following equation:

$$
P_{x i}=R_{x i}-\frac{\sum_{i \in I_{x, y}} \operatorname{sim}(x, y)\left(R_{y i}-R_{i}\right)^{2}}{\sum_{i \in I_{x, y}} \operatorname{sim}(x, y)^{2}} .
$$

Compared with other similarity calculation methods, the Jaccard coefficient is more suitable for dealing with sparse data. However, the Jaccard coefficient does not consider the specific rating values of users for the items, but only whether the users have rated the items, which leads to the accuracy of the similarity calculation results among users. In this regard, we combine the Jaccard coefficient with the modified cosine similarity and use the Jaccard coefficient to improve the modified cosine similarity to solve the problem of accuracy degradation caused by sparse data. The formula of the modified cosine similarity optimized by the Jaccard coefficient is shown below:

$$
\operatorname{sim} \_j \operatorname{arcard}(x, y)=\frac{\sum_{i \in I_{x, y}}\left(R_{x i}-R_{i}\right) \times\left(R_{y i}-R_{i}\right)}{\sqrt{\sum_{i \in I_{x, y}}\left(R_{x i}-R_{i}\right)_{i}^{2} \times\left(R_{y i}-R_{i}\right)_{y}^{2}}} \times \frac{\left|M_{x}^{y} \cup N_{x}^{y}\right|}{\left|M_{x}^{y} \cap N_{x}^{y}\right|} .
$$

The modified cosine similarity is more suitable for situations where users have many common rating items, while the Jaccard coefficient is more suitable for situations where users' ratings are clustered and the rating distance is short. The optimized modified cosine similarity combines the advantages of both and is more widely applicable. The optimized modified cosine similarity not only takes into account the scale of different users' ratings and the influence of users' rating values on the similarity calculation results but also takes into account the user preferences implied by the rating distribution, which increases the accuracy of user similarity information [60] and avoids the influence of data sparsity, thus improving the accuracy of similarity calculation:

$$
w=\left(\sqrt{\frac{\sum_{i \in I_{x, y}}\left(R_{y i}-R_{i}\right)^{2}}{\sum_{i \in I_{x, y}} \operatorname{sim}(x, y)^{2}}}+\sqrt{\frac{\sum_{i \in I_{x, y}}\left(R_{y i}-R_{i}\right)^{2}}{\sum_{i \in I_{x, y}} \operatorname{sim}(y, x)^{2}}}\right) * \sqrt{\frac{\sum_{i \in I_{x, y}}\left(R_{x i}-R_{i}\right) \times\left(R_{y i}-R_{i}\right)}{\sqrt{\sum_{i \in I_{x, y}}\left(R_{x i}-R_{i}\right)_{i}^{2} \times\left(R_{y i}-R_{i}\right)_{y}^{2}}}} .
$$


Further improvements are made to the modified cosine similarity. Since the weight considers the proportion of the common rating items of the users and the high and low values of the users' ratings of the items, introducing weight in the modified cosine similarity will help to further improve the accuracy of the similarity calculation.

$$
\operatorname{new} \_\operatorname{sim}(x, y)=\frac{\sum_{i \in I_{x, y}}\left(R_{x i}-R_{i}\right) \times\left(R_{y i}-R_{i}\right)}{\sqrt{\sum_{i \in I_{x, y}}\left(R_{x i}-R_{i}\right)_{i}^{2} \times\left(R_{y i}-R_{i}\right)_{y}^{2}}} \times \frac{\left|M_{x}^{y} \cup N_{x}^{y}\right|}{\left|M_{x}^{y} \cap N_{x}^{y}\right|} \times w .
$$

Whether it is data mining or neural network model training, data cleaning is quite an important part, the good or bad data cleaning will directly affect the model effect and the conclusion, with the continuous development of computer technology, and in data cleaning, there is a set of more practical process and method. The process is roughly divided into the preprocessing stage, processing missing data, removing data with the wrong format, removing data with wrong logic, etc. In the data preprocessing stage, it is found that there are many problems in the original data; for example, some exercises are not parsed, some exercises have duplicated data, some answers to exercises are written unreasonably, etc. All these problems must be solved in the data cleaning stage. In general, missing value processing can be carried out in the following four steps: determining the range of missing values, removing unwanted fields, completing missing values, and rejudging whether there are still missing values. In the data preprocessing, it is found that the missing part of the exercises is mainly the parsing part, and the parsing is indispensable as an important part of each exercise, so the range of missing values is set as the parsing part of each exercise, and the missing values are made up by imitating the way the parsing of other topics is written; otherwise, the data will be deleted directly.

$$
\begin{aligned}
& \mathrm{SQ}=\left(\frac{\sum_{i=1}^{M} w_{i 1}}{m}, \frac{\sum_{i=1}^{M} w_{i 2}}{m}, \ldots, \frac{\sum_{i=1}^{M} w_{i m}}{m}\right), \\
& \mathrm{ST}=\left(\frac{\mathrm{SQ}_{1}}{m}, \frac{\mathrm{SQ}_{2}}{m}, \ldots, \frac{\mathrm{SQ}_{m}}{m}\right) .
\end{aligned}
$$

A graph convolutional neural network is a neural network model that performs a convolutional operation on a graph. This convolutional operation has many similarities to the convolutional operation in convolutional neural networks, such as weight sharing, local connectivity, and multilayer networks. In the middle school English knowledge graph there are many exercise nodes and interconnected edges; in representing one of the exercise nodes, the model will use its surrounding nodes to represent this node; this is the process of graph convolution in the process of convolution; the features of the exercise nodes around this exercise node will have an impact on the representation of the current exercise node; the essence of this process is feature extraction; as the number of model training continues to increase, deeper features are extracted. A graph convolutional neural network has multiple hidden layers, and in a multilayer graph convolutional neural network, the propagation rules between layers are defined by equation (7).

$$
\begin{gathered}
H_{i}^{j}=\delta\left(D^{1 / 2}\left(A+I_{N}\right) H^{i} W^{j}\right), \\
L=U \nabla U^{T} .
\end{gathered}
$$

Multiple convolutional layers can be stacked in the neural network, and each convolutional layer is composed of the above equation (8). As the number of convolutional layers increases, the parameters in the model also increase, which leads to the emergence of the problem of slow computation and overfitting of the model. To prevent the above problems, the number of convolutional layers is set to 2 in this paper, and its forward propagation model can be simply written as equation (9).

$$
Z=\operatorname{soft} \max \left(A \operatorname{RELU}\left(A H^{i} W^{j}\right) W^{1}\right) \text {. }
$$

In generating the middle school English knowledge graph, whether there is a connection between two nodes is determined by the cosine similarity of the two exercise sentence vectors, and when the thresholds are different, the number of edges in the generated knowledge graph is different and the model results are different.

2.2. English Learning Platform Design. The overall goal of the cybersecurity self-directed learning platform is not only to provide traditional online learning functions, but also to provide personalized learning recommendation services, i.e., to collect and organize learners' basic personal information, rating information, and historical learning behaviour records, to realize a personalized learning recommendation service for cybersecurity self-directed learning by using a Novel User-based collaborative filtering recommendation algorithm (NUCF) proposed in Chapter 3. NUCF is proposed in Chapter 3 to realize a personalized learning recommendation service for cybersecurity self-directed learning. Compared with the user's online learning experience, the platform pursues the user's personalized service experience more. In the current Internet information era, there are increasing online learning platforms for cybersecurity, which share a large amount of learning resources and data, but they are unable to recommend to users the resources they want from the huge amount of cybersecurityrelated learning data. This is the main goal of the cybersecurity self-directed learning platform developed in this paper [24]. The cybersecurity self-directed learning platform developed in this paper will break the limitation that traditional cybersecurity online learning platforms are only used for sharing and using learning materials. By introducing 
efficient personalized recommendation algorithms, it helps clueless learners to find a set of learning routes and related learning materials suitable for themselves among the huge data of learning materials.

The network security independent learning platform has four main functional modules: information management module, user management module, personalized learning recommendation module, and main learning module. Each module is further divided into different functional submodules, which collaborate, thus making the whole platform efficient and concise, reducing users' unnecessary searching and searching time and realizing efficient learning, as shown in Figure 2.

Before building the network security independent learning platform, the most critical step is the research and analysis of the platform requirements. The platform requirement research and analysis are the key to reflect the value of this platform. The demand research and analysis of this platform considers the influence of many specific factors in the real environment, such as the necessity of platform development, technical support for development, market competitiveness, development and maintenance costs, and other factors. We analysed the requirements from all aspects to lay the foundation for the design and development of the platform and reduce unnecessary troubles in the development process. Before the formal development work was carried out, we also conducted a series of assessments on the feasibility of the network security independent learning platform and other indicators, and the assessment results were that the platform design, development, operation, and maintenance needs can be completed through technical means; also, the design, development, operation, and maintenance of this platform have economic feasibility and legal feasibility. In the following, the feasibility of this platform will be analysed from three aspects: technical feasibility, economic feasibility, and legal feasibility.

The information management module is set for the platform administrators. This module helps platform administrators to maintain and manage the platform better so that the platform can run stably for a long time and can be expanded effectively. The module updates the learning resources of the platform so that the platform can be maintained and the information can be updated so that the platform can be most effective. The module uploads relevant book information to the database and queries, updates, and deletes book information. Book information specifically includes the book title, book type, book difficulty, book description, etc. After the user logs in, the platform will recommend relevant book information for him/ her. The design of the study route recommendation function is like the study resource recommendation, with slight differences. If the user has previously made a study route, the study route table will be read and passed to the user. If the current user has not developed a learning route, the NUCF algorithm is utilized to recommend a personalized learning route for the user. Also, when the user's ability level reaches a certain standard, the platform will determine whether the user will get into the next stage of learning, to readjust the learning route to suit the current user's level and provide personalized recommendation services for registered users.
This module is an auxiliary module of the platform, and the user learning data collection mainly serves the personalized learning recommendation module. The module will record, collect, and format the data and related behaviours of users when they use the platform and store them separately by different users. When users use the function of the personalized learning recommendation module, the data collected by this module will be used as the input of the NUCF algorithm; i.e., it provides effective data support for the NUCF algorithm, as shown in Figure 3.

The adaptive learning system is applied to the pc terminal, and the main user groups of the system are mainly four categories: students, teachers, faculty, and administrators. After entering the system, students can browse the course information, exam list, system announcement, etc., and choose suitable and preferred resources for learning. After learning proceeds to a certain stage, they can choose to enroll in the exam resources released by the course teachers, and the system performs all-round analysis and interpretation of the exam results according to the students' answers to help students understand their conditions and clarify their learning goals and learning tasks. By accurately recording students' learning behaviours (resources clicked and time points, doing records and accuracy rate, test questions containing knowledge, resource return rate, etc.) to obtain each student's exclusive learning log, analysis system makes scientific and intelligent analysis, to provide personalized exams for each student. Teacher users can monitor and manage teaching resources after logging into the system and at the same time realize the functions of creating courses and uploading courseware resources and test paper resources, submit the uploaded teaching resources to faculty affairs for review, and then release them to the system platform if they pass the review and return the teaching resources that fail to pass the review to teacher users for further modification and adjustment, when teachers teach courses for examinations. Also, teachers have the right to monitor the analysis of students' learning ability, so that they can view the analysis of students' exams and cognitive diagnosis of each student after each exam, which is convenient for timely adjustment of teaching progress and learning content.

Academic affairs are responsible for the audit and management of system education resources and system user viewing and management, as well as the release of teaching resources and announcement information, audit the uploaded teaching resources and release them to the system platform, and return them to teacher users if they do not pass the audit, to ensure the high quality of system platform resources and timely release of announcement information related to education teaching, course resources, and examination information. It is convenient for students and teachers to understand the latest courses and other related contents; the statistical analysis of the registration situation of courses and exams is displayed, which can understand the learning status of students and the popularity of the courses, to prepare for the development of the next phase of course plans. The administrator is the manager and maintainer of the adaptive learning system, assigning appropriate permissions to the users of the system and managing all the 


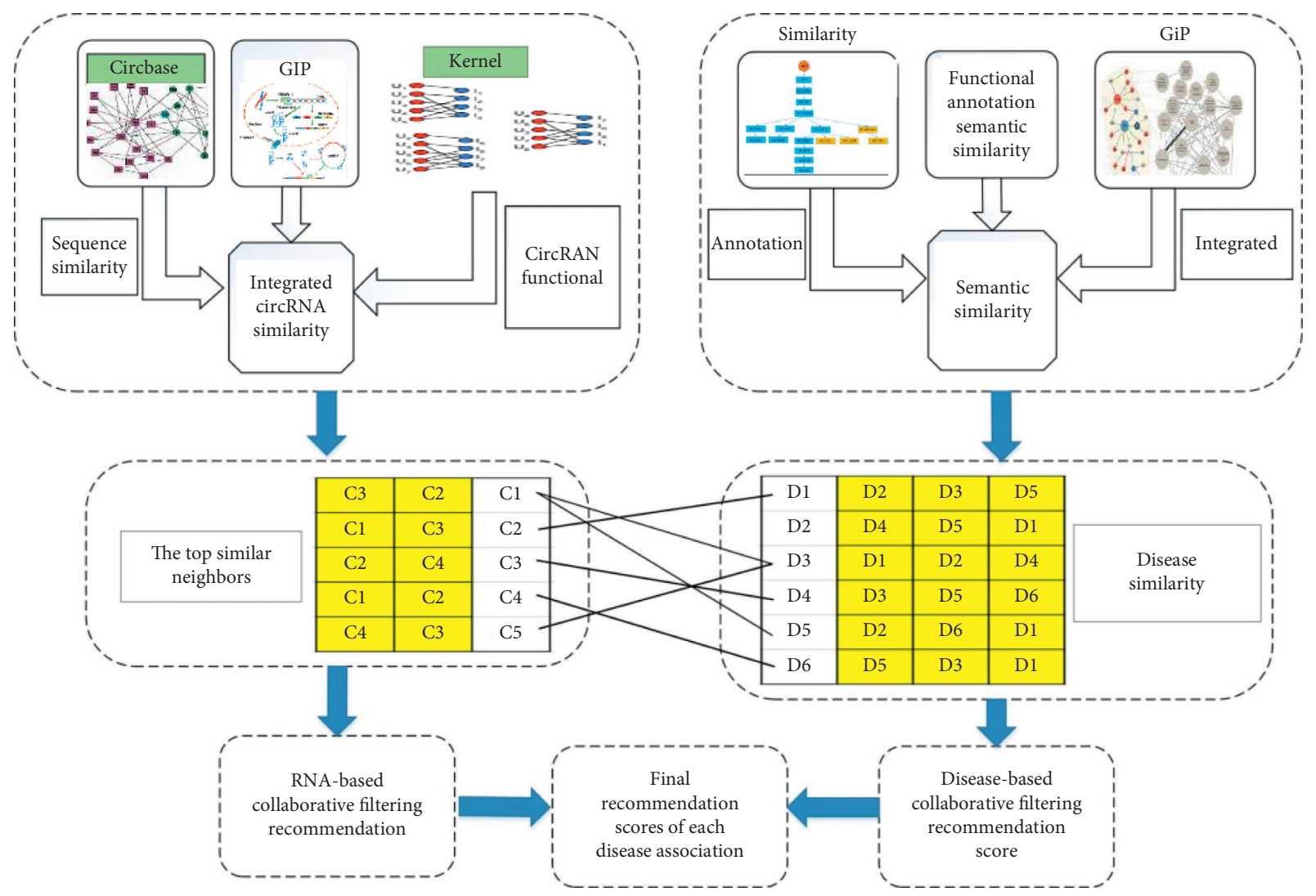

FIGURE 1: Improved collaborative filtering algorithm.

resources of the system to ensure the continuous and safe operation of the system and provide services.

2.3. Performance Analysis Design. The teaching targets are sophomore students, whose intellectual development tends to mature and their cognitive ability has further improved. Students' ability to acquire information in English, internalize information, analyse information, and solve problems has been improved, and the pretest questionnaire shows that students in this class want to improve in vocabulary and grammar teaching, so the teaching design should pay special attention to improving students' ability in vocabulary and grammar. When analysed in terms of learners' learning styles, most students are still willing to accept the teaching style of blended learning mode. Prelearning before class is an important part of blended learning, in which students complete independent learning through the prelearning materials provided by Sequoia Smarting and test the effect of self-learning through prelearning tests. The preliminary understanding of their learning deficiencies enables more targeted classroom learning, which is conducive to the development of teachers' classroom teaching and the expansion of their knowledgeability in classroom lectures, as shown in Figure 4.

At the end of each lesson, the platform will collect students' evaluation of the teacher. The evaluation will be used for the teacher information display page display, and the rating data can also be used as calculation data when the teacher is recommended. Also, this evaluation contains feedback on the quality of the lesson, which is used for the subsequent optimization of the platform content. This module will introduce the process of student evaluation of a lesson. To make the personalized learning path generation more reasonable, it is necessary to generate a difficulty value for each exercise and to rank the exercises according to their difficulty value when generating the personalized learning path. There is no unified standard or criteria for the assessment of the difficulty of the exercises. In this paper, the difficulty of the exercises is examined in three aspects: the presence of superlatives in the exercises, the similarity of the answers, and the number of knowledge points examined in the exercises, where the similarity of vocabulary is divided into near-synonyms and morphological similarities. For essay and translation questions, it was not possible to assess the difficulty level based on the above aspects. For these questions, the difficulty level was set as the average of the difficulty level of all the exercises.

In this paper, we crawled some of the exercise data with difficulty values from the Internet, analysed the batch of exercises from the above three aspects respectively, summarized the conclusions in Table 1 for different assessment perspectives, and corresponded the three difficulties of easy, medium, and difficult to the scores 1,2 , and 3 , respectively.

After the personalized knowledge graph is generated, all the exercise nodes in the knowledge graph are labelled, and the similarity between the student's work and the exercise nodes is used in the personalized knowledge graph. The 


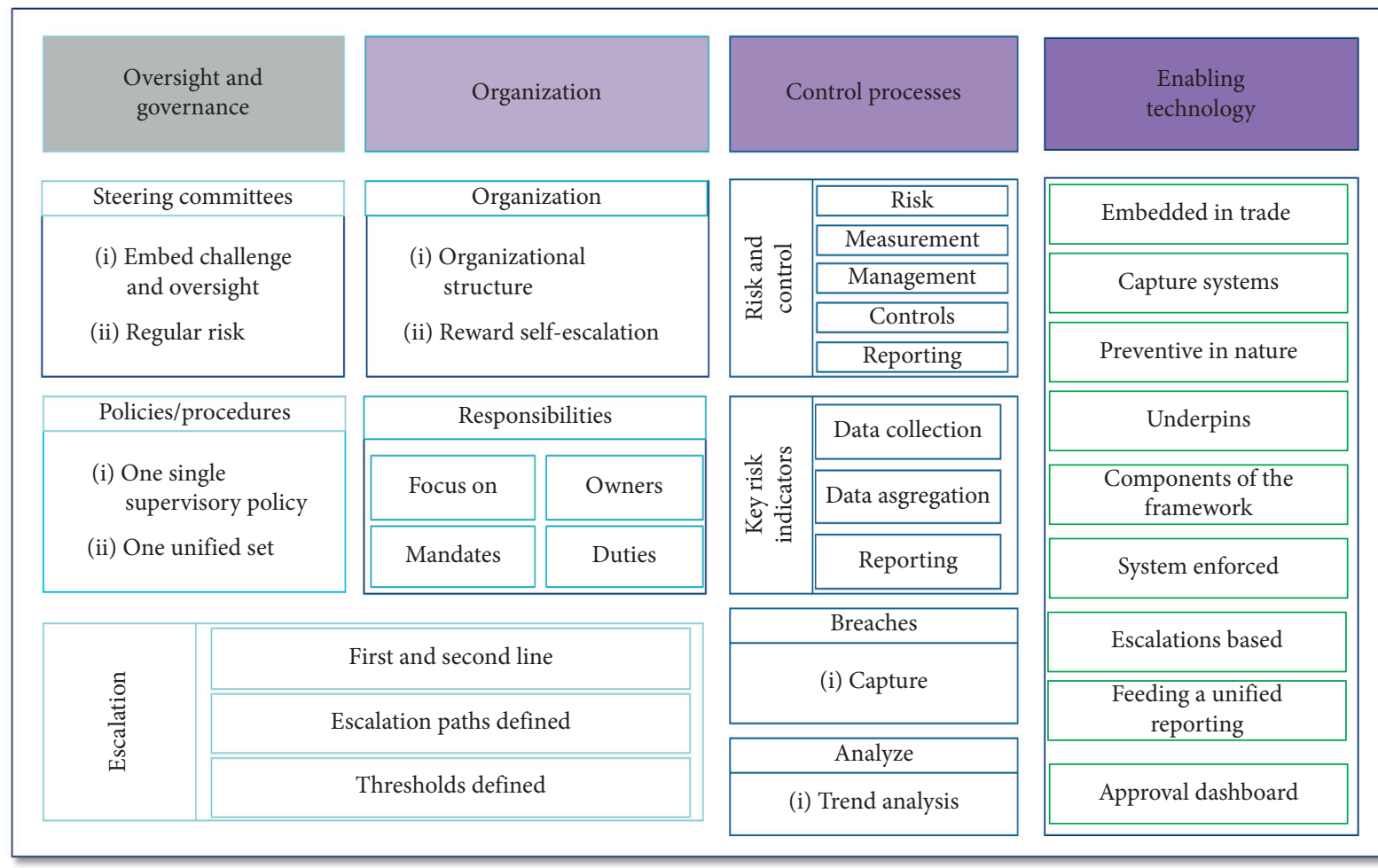

The overall architecture of the learning platform

Figure 2: The overall architecture of the learning platform.

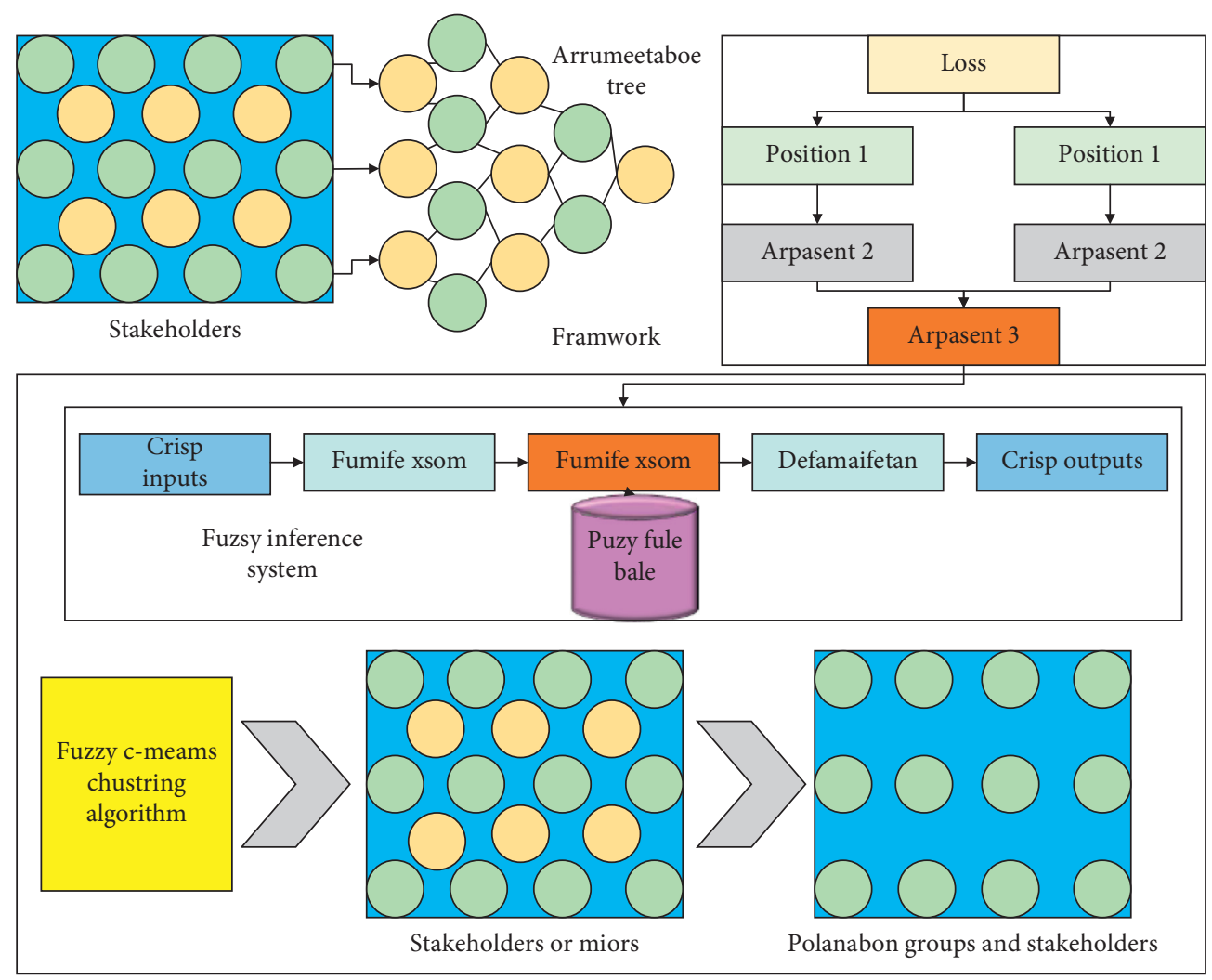

FIgURE 3: Database table E-R diagram. 
exercises that make up the personalized learning path are selected. After that, personalized learning paths are generated according to the difficulty level of the exercises. In this paper, we use the similarity between the exercise nodes to select the exercises and borrow the ideas of prim algorithm and mask algorithm. When using the prim algorithm to generate the minimum spanning tree, the vertices are first divided into two categories. One is included in the tree in the process of finding, and the other is not yet included in the tree; in the generation of the minimum spanning tree, the starting state of all vertices is classified as the second category [25]. After that, any vertex is selected as the starting point and moved from the second class to the first class; then the vertex with the smallest weight between the vertices in the second class and the first class is found and moved from the second class to the first class, until there are no vertices in the second class. The Kruskal algorithm was issued by Joseph Kruskal in 1956 and fully embodies the idea of a greedy algorithm. Kruskal algorithm in generating the minimum spanning tree first constructs a subgraph containing only $n$ vertices, and the set of edges is empty. The vertices in the subgraph are regarded as the first thing you need to do is to select an edge with the smallest weight from the set of edges; if the two vertices of the edge belong to different trees, it will be added to the subgraph, that is, the two trees into one tree, and vice versa; if the two vertices of the edge fall on the same tree, it is not desirable and should take the next edge with the smallest weight and try again, until the subgraph contains $n-1$ edges. In this paper, the exercise nodes are selected from multiple nodes and the neighbouring nodes with the greatest similarity to these nodes are found, and the similarity between the exercise nodes can be regarded as the weights on the edges. The exercise paths generated in this paper require that the similarity between the exercise nodes connected by edges in the path is the greatest, which is consistent with the idea of generating a minimum spanning tree. Also, it is not required in this paper that all the selected exercise nodes are connected.

\section{Analysis of Results}

3.1. Analysis of Filtering Algorithm Results. Students' learning process and practice process are essentially the learning and consolidation of knowledge points. Based on the analysis of students' learning logs, we can construct students' exclusive ability portraits and recommend and practice learning resources according to their abilities, which can achieve more efficient and targeted learning. The system precisely records the process of students doing the questions, including the steps they took, the knowledge points involved, and the time when the actions were taken, etc. Through the analysis of the learning logs, students can pinpoint the reasons for their practice errors and truly master the unskilled knowledge points. Transforming the logs of students' interactions with test questions into the assessment of students' ability based on knowledge points helps students and teachers understand their learning status and carry out timely learning and consolidation. After obtaining the students' ability portrait, the learning resource recommendation module adopts the personalized test resource recommendation method based on deep learning to recommend personalized test questions and further evaluates the recommendation results to ensure that the difficulty of the recommended resources is in a reasonable and appropriate range, so that the recommended results are not too easy, as shown in Figure 5.

The evaluation subjects of this learning model are diversified, and the evaluation mainly consists of students' evaluation, group members' evaluation, teacher's evaluation of students in the class, and the Sequoia Smart Platform's allaround recording of data of the whole process. The data recorded by the new lecture mode include the efficiency of students in the learning class, the correct rate of pretest and test questions in class, etc. The above data can be fed back to students in real-time in the Sequoia Smart Platform to achieve the function of reminding and motivating students, as shown in Figure 6.

For students, after the class is finished, the teacher will have the exercises pushed, and after the students finish, the teacher can make an evaluation according to the students' completion, and the platform will also reward different students with corresponding gold coins according to their completion. According to the previous teaching model, the teacher's evaluation of students is essential. Therefore, the blended learning model can take this advantage and combine the teacher's evaluation of students' class participation, answering questions positively and correctly, written assignments, test scores, etc. The evaluation of this model belongs to process evaluation, in which the quantitative evaluation is mainly conducted and recorded by the data of Sequoia Smart Platform, and the statistical objects include the teacher's evaluation of students' learning status and learning effect.

The qualitative evaluation includes the evaluation among group members and the teacher's evaluation of students' performance during the lecture. The experiment took three months, and by comparing the English test scores of the students in the two classes before and after the experiment, the statistics were tabulated to observe the changes produced by the two scores before and after the experiment and to observe whether the changes were significant and to what extent they had changed. It is in the range of moderate correlation and its sig value is also less than 0.5 , which is within the norms of the T-test. According to Figure 7, the sig value of the control class was equal to 0.105 while the sign of the experimental class was equal to 0.001 . The control class was greater than the standard value of 0.05 while the experimental class was less than 0.05 , which indicates that there was a significant difference between the pre- and posttest scores of the experimental class, which means that the hybrid learning model based on the intelligent platform had a significant impact on the students' learning outcomes.

3.2. Analysis of Platform Optimization Results. After three months of blended learning, $97.9 \%$ of the students gave feedback that this learning model has improved their interest in learning and they like the class more, and this kind of class 


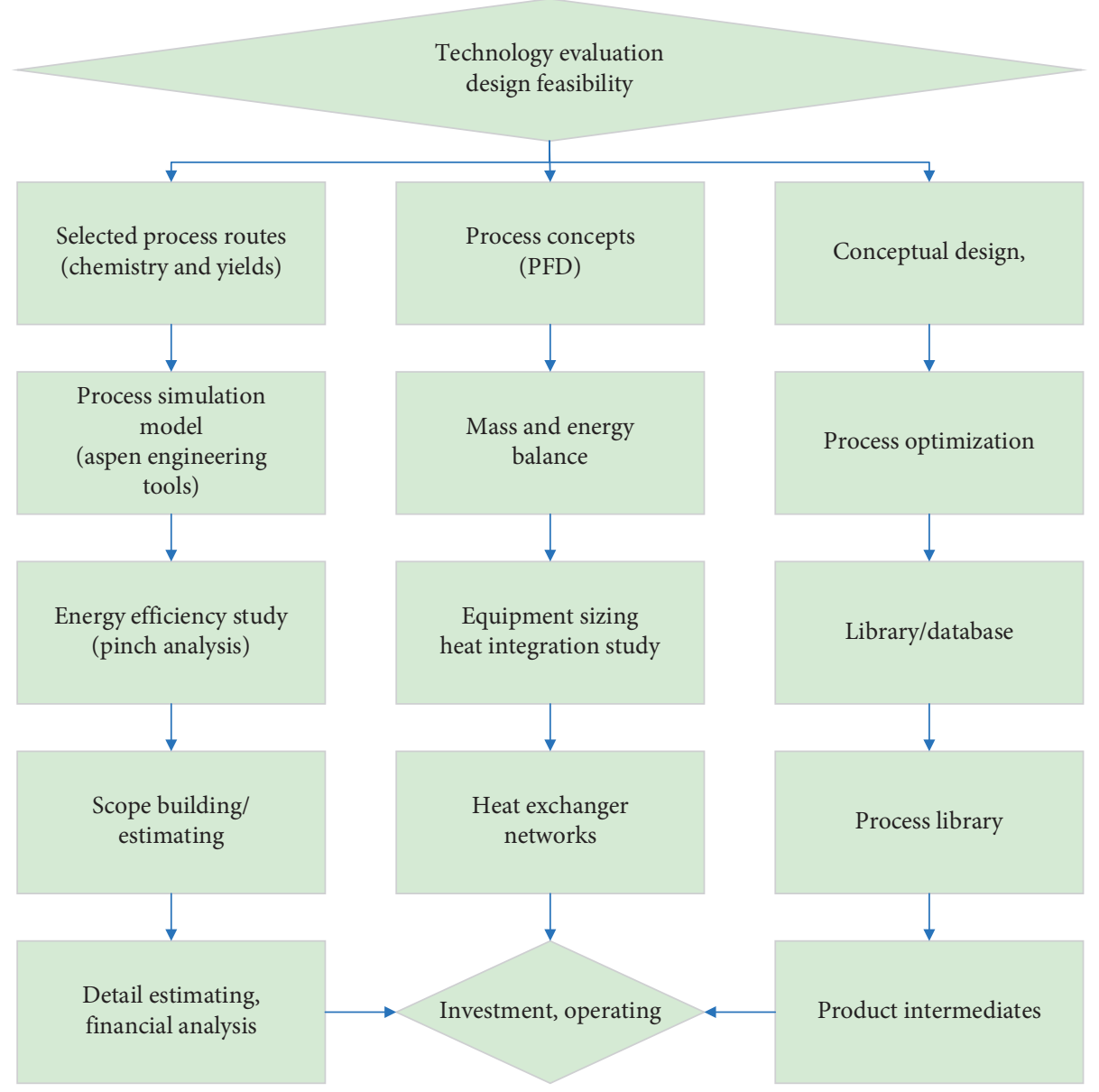

Figure 4: Performance design framework.

TABLe 1: Difficulty and ease of analysis.

\begin{tabular}{|c|c|c|c|}
\hline Evaluation angle & Problem situation & Degree of difficulty & Points \\
\hline \multirow{3}{*}{ Super vocabulary } & Nonsuperclass vocabulary & Simple & 1 \\
\hline & Have 1 or 2 superclass vocabulary & Medium & 2 \\
\hline & More than two superclass vocabulary & Difficult & 3 \\
\hline \multirow{3}{*}{ Answer similarity } & The answers are not similar & Simple & 4 \\
\hline & Both answers are similar & Medium & 5 \\
\hline & More than three answers are similar & Difficult & 7 \\
\hline \multirow{3}{*}{ Survey the number of knowledge points } & Examine a knowledge point & Simple & 2 \\
\hline & Investigate two knowledge points & Medium & 3 \\
\hline & Investigate more than two knowledge points & Difficult & 2 \\
\hline
\end{tabular}

can make students understand the new knowledge faster, which means that this blended learning mode helps to improve students' interest in learning; $93.7 \%$ of the students think that their learning initiative has improved; $91.7 \%$ of the students have improved their self-learning ability; $93.8 \%$ of the students think that they can manage their learning time consciously and playfully after the smart platform, and they can form good learning habits. $93.8 \%$ of the students think that they can manage their study time consciously and systematically after learning on the intelligent platform and can form good study habits; $91.7 \%$ of the students are more willing to carry out cooperative learning with their classmates than before. The comparison of the before and after questionnaires of the above questions shows that using blended learning mode can improve students' learning ability, as shown in Figure 8.

After the three-month teaching experiment, to have a deeper understanding of students' knowledge and skills level, students' classroom satisfaction, students' learning ability, and students' acceptance of the blended learning model were evaluated. After the teaching experiment, an interview was conducted in November 2018 with a random 


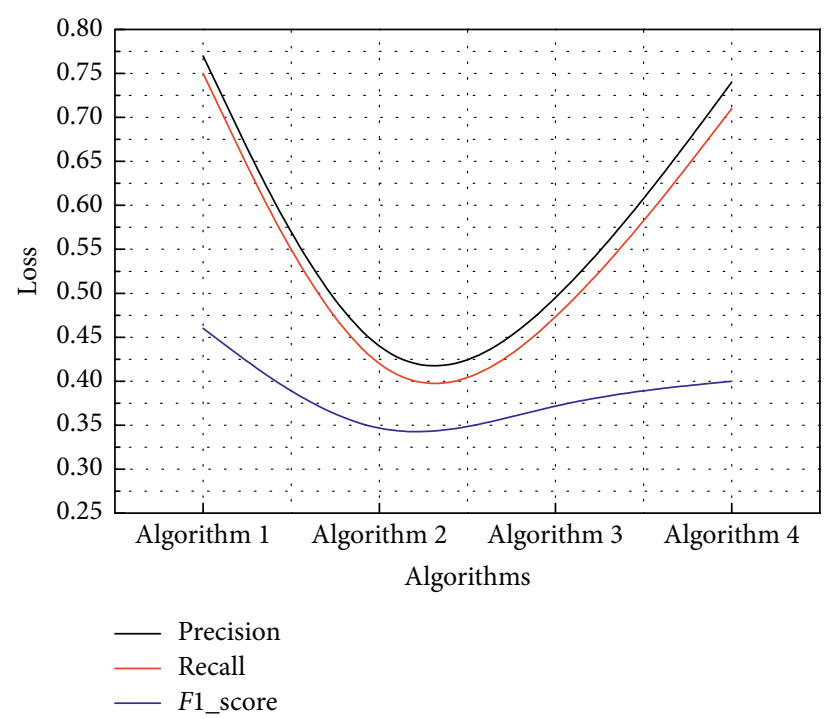

Figure 5: Performance comparison chart.

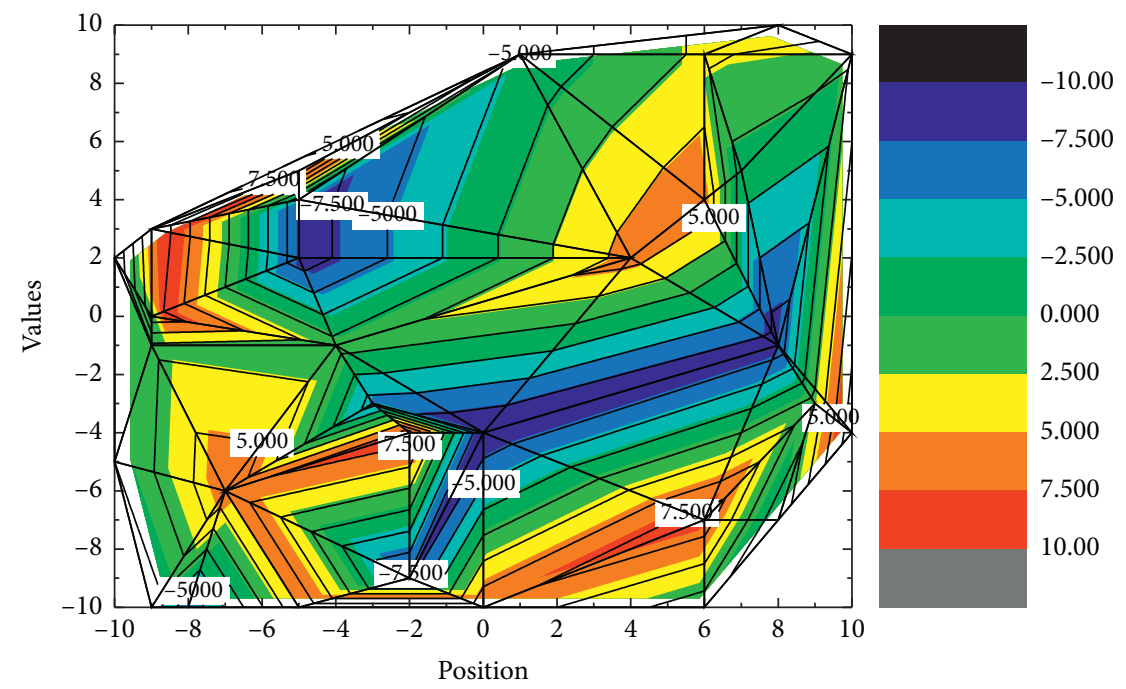

Figure 6: Group statistics and independent sample $t$-test results.

sample of four students from the subject class and the teacher of the class based on the postexperimental questionnaire distributed. The advantages of interviews are that they allow face-to-face communication with the study subjects, clearer questions, more specific views obtained from the subjects about the survey, better understanding of students' specific ideas and perspectives, and more comprehensive feedback on the issues. The environment and atmosphere of the interviews were relaxed, stress-free, and relatively authentic, and the entire interview process was conducted using an audio recording tool for later data compilation, as shown in Figure 9.

The academic affairs page facilitates the management of faculty information by faculty members, including teachers registered under that faculty and the courses, tests, and other resources they publish, students enrolled in courses or exams, and exam score management. The main function of the backend part of the adaptive learning system is to realize the management of system resources, and the user groups targeted are teachers, faculty members, and system administrators. Among them, teacher users realize the management of course resources (including courses and test questions); academic affairs users realize the management of teachers, students, course resources, fee payment resources, etc.; administrator users realize the management of system users and corresponding rights, as well as the maintenance of resources, as shown in Figure 10.

Module testing is the testing of the system, the smallest design unit. Firstly, it is compiled and checked to modify syntax errors; then, regarding the detailed design document of the adaptive learning system, the module interface, execution path, error handling path, and boundary conditions 

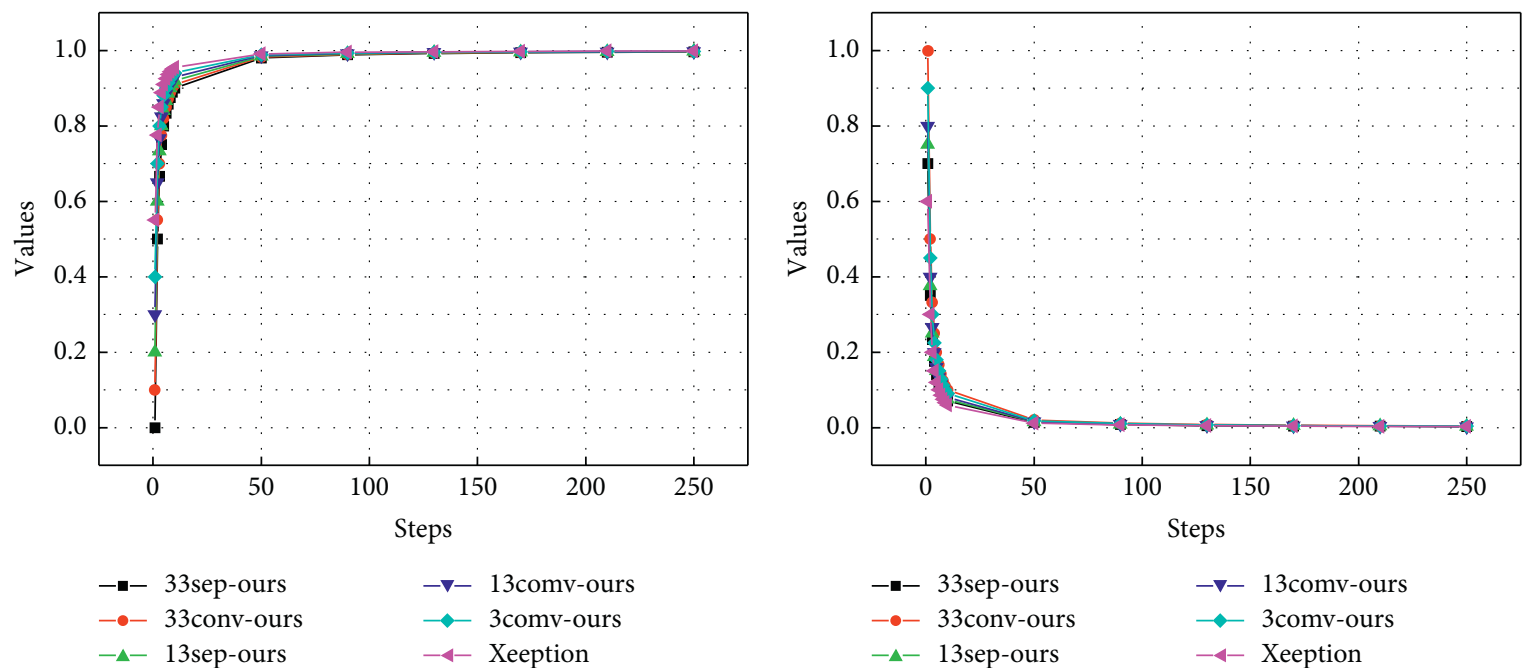

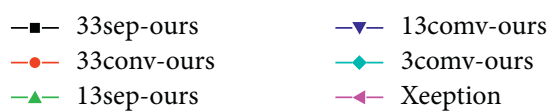

(a)

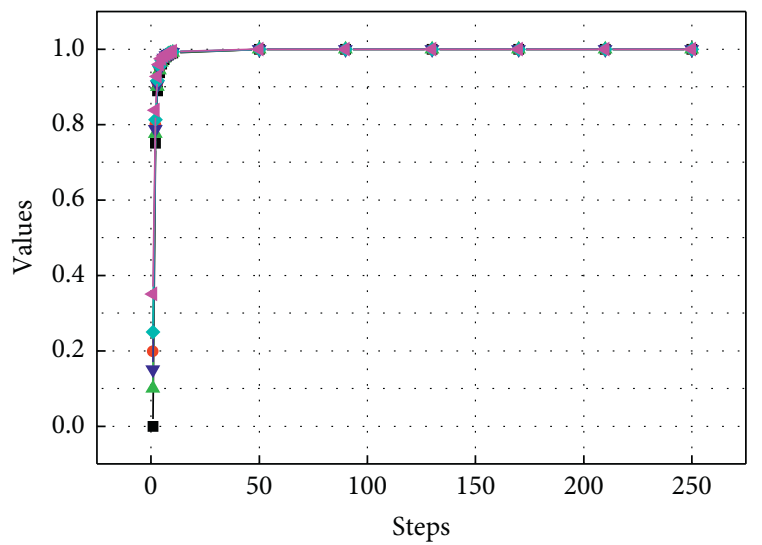

$-\nabla-13$ comv-ours

$\multimap$ 3comv-ours

-4 Xeeption

(c)

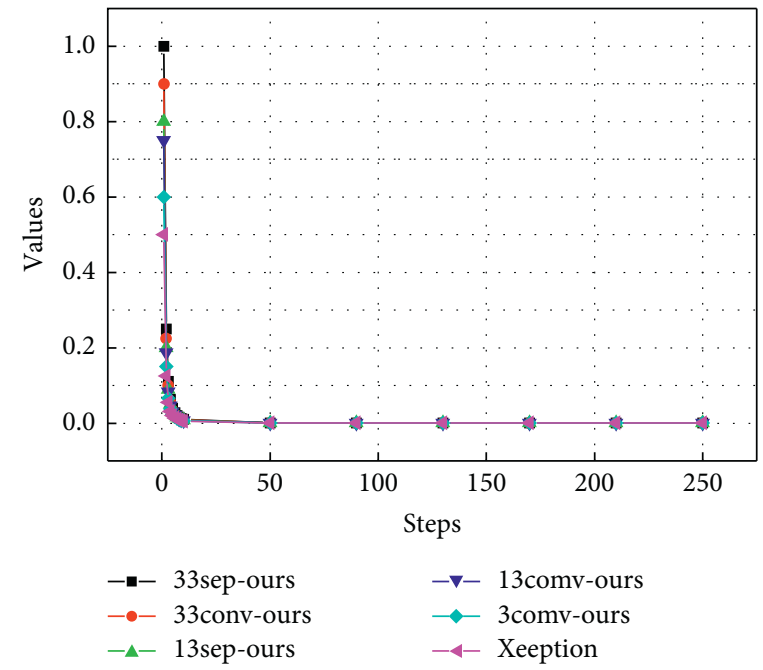

(d)

Figure 7: Sample statistics.

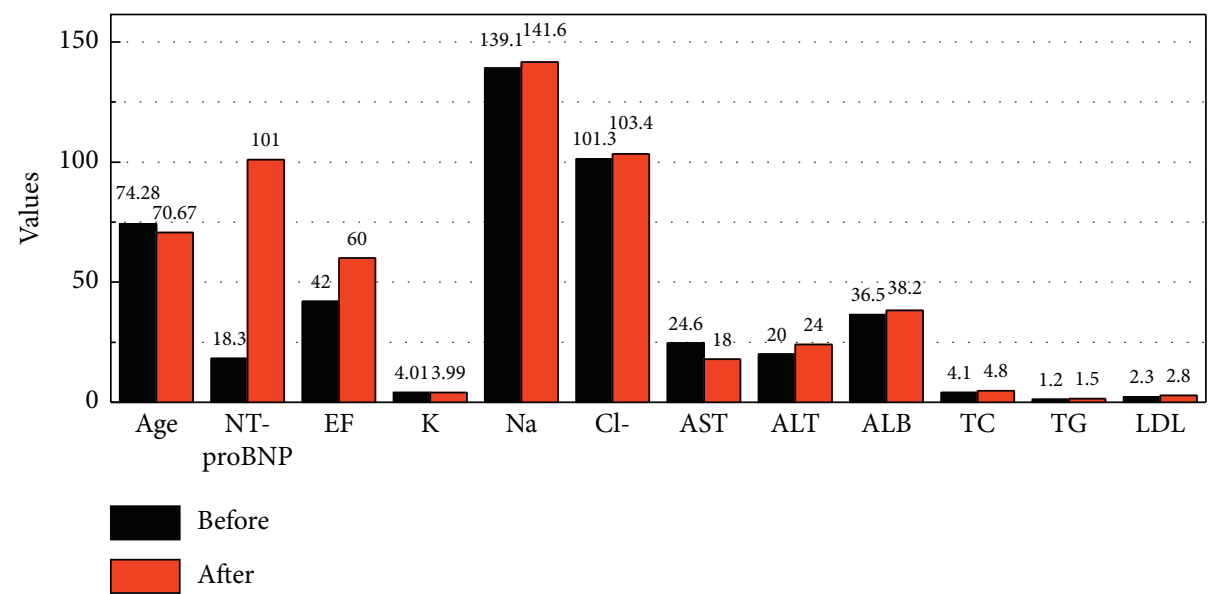

(a)

FIgure 8: Continued. 


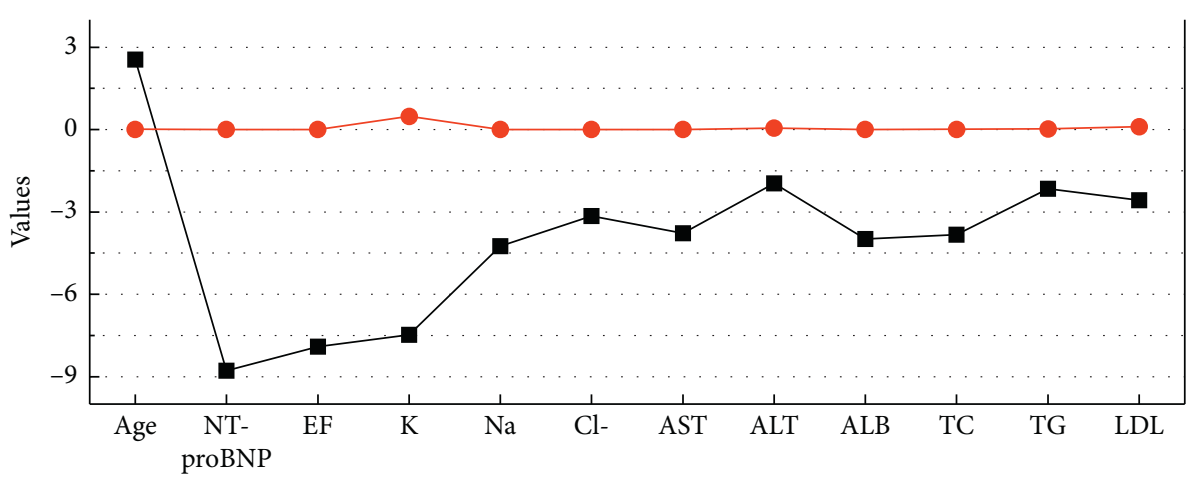

(b)

Figure 8: Performance analysis.

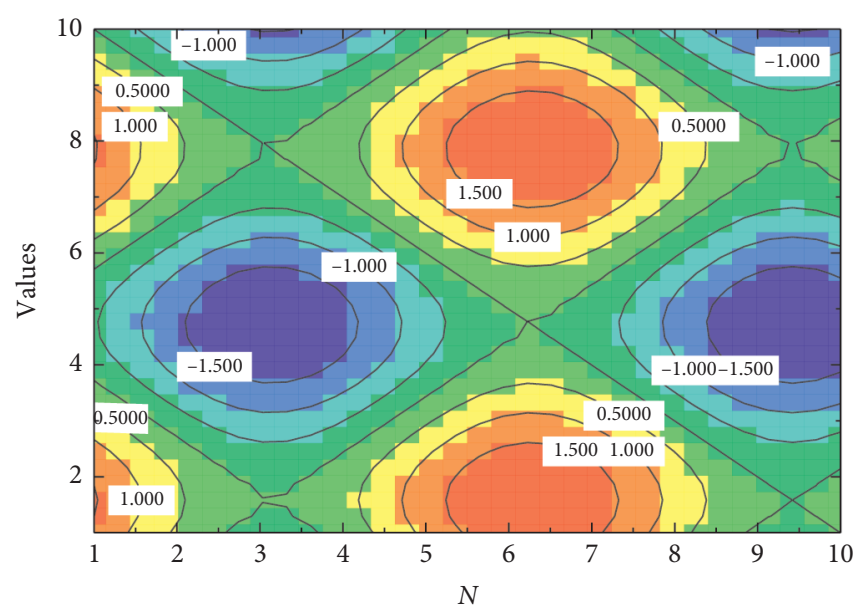

Figure 9: Performance results.

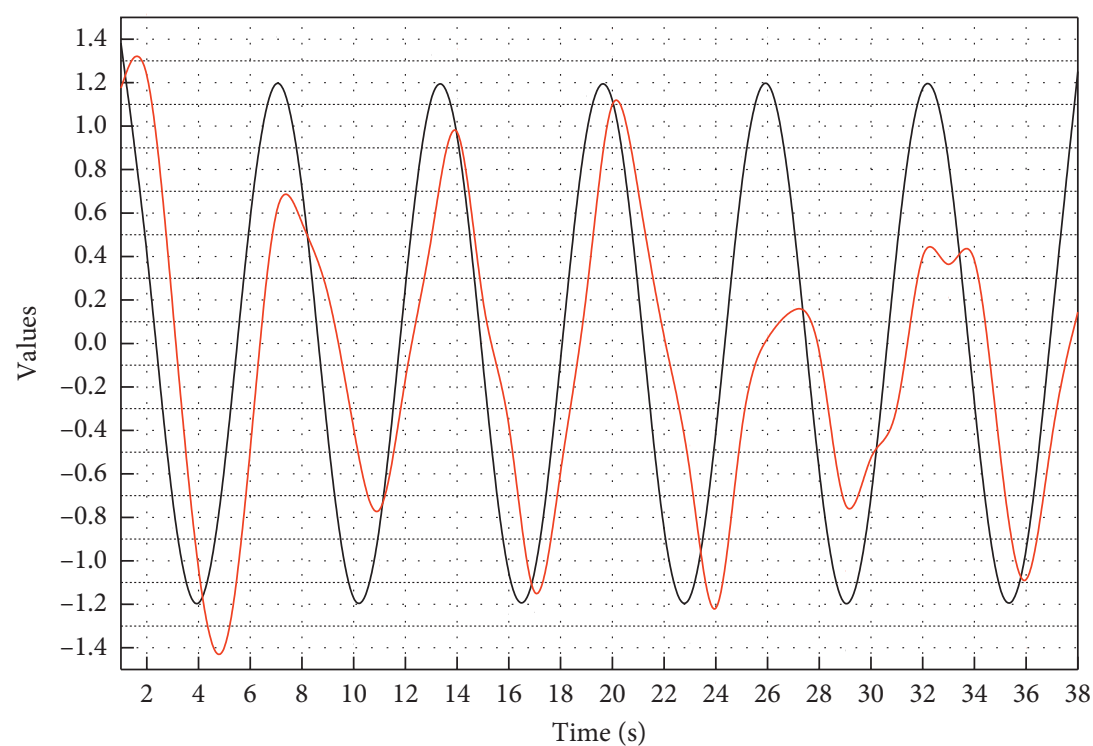

Before optimization

After optimization

Figure 10: Performance test results analysis. 
of each functional module of the system are tested and evaluated; finally, the errors checked are corrected to ensure the normal operation of each module. System testing of the integrated system is carried out with the requirements definition at the beginning of the system design as a reference to ensure that the implemented functions and performance meet the specified requirements. The adaptive learning system is to guarantee that administrators, faculty, teachers, and student groups work together to provide students with specific functions such as online learning, learning analysis, and personalized testing. After system testing of adaptive learning under the guidance of adaptive learning system design in Chapter 2, it is ensured that this system can meet the expected requirements and operate normally. The role of the acceptance test is to show that the developed system program can work according to the expected standard. However, due to the limited development environment of the system, this part of the testing work was participated by the students in the lab on behalf of the system, and the tests included some tests related to the user's usage steps and performance functions of particular interest to the user, while the purely technical tests were not conducted. The system accomplished the target work, but there is still room for improvement in the smoothness of the system operation.

\section{Conclusion}

The current social technology is changing very fast and artificial intelligence is slowly being combined with education, which requires teachers in the new era to constantly update and explore innovative teaching methods to improve teaching effectiveness. The blended teaching mode based on an intelligent platform is a new teaching model, which is a combination of technology and modern teaching. There is still relatively little use of the blended learning model in high school English teaching, so the author's research is to build a different model for the combination of those. This paper designs a hybrid online and offline teaching model based on online learning space and applies it to the teaching of higher education courses and tests the application effect of online learning space for college students to improve the learning initiative and learning effect of college students in online learning space and to promote the reform of higher education teaching. The overall architecture design, functional module design, and database design of the platform were carried out. Through the functional module design, the entire platform is decomposed into several submodules with low coupling and high cohesion, and we have analysed the design method of each submodule in detail. Different modules collaborate to make the platform run and operate concisely and efficiently, thus reducing users' unnecessary searching and searching time and achieving efficient learning.

\section{Data Availability}

The data used to support the findings of this study are available from the corresponding author upon request.

\section{Conflicts of Interest}

The authors declare that they have no known competing financial interests or personal relationships that could have appeared to influence the work reported in this paper.

\section{References}

[1] S. Zou, "Designing and practice of a college english teaching platform based on artificial intelligence," Journal of Computational and Theoretical Nanoscience, vol. 14, no. 1, pp. 104-108, 2017.

[2] Y. Bin and D. Mandal, "English teaching practice based on artificial intelligence technology," Journal of Intelligent \& Fuzzy Systems, vol. 37, no. 3, pp. 3381-3391, 2019.

[3] W. Jun, "College english teaching mode based on intelligent robot," International Journal of Continuing Engineering Education and Life Long Learning, vol. 29, no. 1-2, pp. 33-43, 2019.

[4] L. Cheng and L. Zhenhua, "Research on smart mobile classroom design from the perspective of game learningtaking preschool education major of distance education as an example," Education Journal, vol. 8, no. 6, pp. 349-353, 2019.

[5] S. Dai, "ARS interactive teaching mode for financial accounting course based on smart classroom," International Journal of Emerging Technologies in Learning (iJET), vol. 14, no. 3, pp. 38-50, 2019.

[6] F. Kong, J. Li, and Y. Wang, "Human-computer interactive teaching model based on fuzzy set and BP neural network," Journal of Intelligent \& Fuzzy Systems, vol. 37, no. 1, pp. 103-113, 2019.

[7] J. Kong, "Innovative applications mode of network learning space in exercise physiology based on ubiquitous learning," International Journal of Emerging Technologies in Learning (iJET), vol. 14, no. 4, pp. 113-126, 2019.

[8] L. Zhao, L. Chen, Q. Liu, M. Zhang, and H. Copland, “Artificial intelligence-based platform for online teaching management systems," Journal of Intelligent \& Fuzzy Systems, vol. 37, no. 1, pp. 45-51, 2019.

[9] N. Zhao, X. Zhou, X. Zhou, B. Liu, and W. Liu, "Guiding teaching strategies with the education platform during the COVID-19 epidemic: taking guiyang no. 1 middle school teaching practice as an example," Science Insights Education Frontiers, vol. 5, no. 2, pp. 531-539, 2020.

[10] A. Alam, "Possibilities and challenges of compounding artificial intelligence in India's educational landscape," International Journal of Advanced Science and Technology, vol. 29, no. 5, pp. 5077-5094, 2020.

[11] C. Shen, "MOOC teaching mode of news transmission based on network audio data decoding technology," International Journal of Emerging Technologies in Learning (iJET), vol. 13, no. 6, pp. 43-55, 2018.

[12] S. Li, "Innovations in Chinese engineering education with digital technologies: a brief review of recent advances," Computer Applications in Engineering Education, vol. 26, no. 5, pp. 1081-1088, 2018.

[13] D. Liu and Q. Hu, "Practice of China's new high-level university of science and engineering's international development: a case study of dongguan university of technology," Region-Educational Research and Reviews, vol. 1, no. 2, pp. 28-33, 2020.

[14] J.-F. Han and P. Lee, "The college english teaching mode for nutrition based on flipped classroom," Journal of 
Computational and Theoretical Nanoscience, vol. 13, no. 12, pp. 9258-9263, 2016.

[15] J.-H. Fan, "On computer and foreign language teaching and learning in big data era," International Journal of Emerging Technologies in Learning (iJET), vol. 13, no. 5, pp. 236-245, 2018.

[16] F. Qiao and H. Wang, "Mobile interactive translation teaching model based on "Internet+"” EURASIA Journal of Mathematics, Science and Technology Education, vol. 13, no. 10, pp. 6705-6714, 2017.

[17] L. H. Li, "Design of college english process evaluation system based on data mining technology and Internet of Things," International Journal of Data Warehousing and Mining (IJDWM), vol. 16, no. 2, pp. 18-33, 2020.

[18] D. Zou, H. Xie, and F. L. Wang, "Future trends and research issues of technology-enhanced language learning: a technological perspective," Knowledge Management \& E-Learning: An International Journal, vol. 10, no. 4, pp. 426-440, 2018.

[19] G. J. Hwang and Q. K. Fu, "Advancement and research trends of smart learning environments in the mobile era," International Journal of Mobile Learning and Organisation, vol. 14, no. 1 , pp. 114-129, 2020.

[20] C. Shuo and C. Xiao, "The construction of internet + piano intelligent network teaching system model," Journal of Intelligent \& Fuzzy Systems, vol. 37, no. 5, pp. 5819-5827, 2019.

[21] R. Liang, "Analysis of the influence of multimedia network hybrid teaching method on college students' learning ability in physical education," International Journal of Continuing Engineering Education and Life Long Learning, vol. 29, no. 1-2, pp. 101-112, 2019.

[22] W. Xiaoning, "Cultivating online English learner autonomy in internet plus era: a DST perspective," Studies in Literature and Language, vol. 13, no. 5, pp. 14-19, 2016.

[23] N. S. Chen, I. L. Cheng, and S. W. Chew, "Evolution is not enough: revolutionizing current learning environments to smart learning environments," International Journal of Artificial Intelligence in Education, vol. 26, no. 2, pp. 561-581, 2016.

[24] M. Ivanova, "eLearning informatics: from automation of educational activities to intelligent solutions building," Informatics in Education, vol. 19, no. 2, pp. 257-282, 2020.

[25] J.-Q. Li, F. R. Yu, G. Deng, C. Luo, Z. Ming, and Q. Yan, "Industrial internet: a survey on the enabling technologies, applications, and challenges," IEEE Communications Surveys \& Tutorials, vol. 19, no. 3, pp. 1504-1526, 2017. 